\title{
SEMIGROUP RINGS AND SEMILATTICE SUMS OF RINGS
}

\author{
JULIAN WEISSGLASS ${ }^{1}$
}

To Harvey Jackins

\begin{abstract}
A generalization of the concept of a decomposition of a ring into a direct sum of ideals is introduced. The question of semisimplicity of the ring in terms of the semisimplicity of its summands is investigated. The results are applied to semigroup rings.
\end{abstract}

1. Introduction. Many substantial results in the theory of rings either concern themselves with or use a decomposition of the ring into a direct sum of ideals. In this paper we introduce a generalization of direct sum decompositions. A ring is a supplementary semilattice sum of subrings if as an additive abelian group it is a direct sum of the additive groups of the subrings and if the subrings multiply in a certain natural way. We begin the investigation of semilattice sum decompositions by showing that in certain cases the semisimplicity of the subrings implies the semisimplicity of the ring. The investigation is carried out in the context of $\pi$-semisimplicity where $\pi$ is a hereditary, homomorphic invariant property of rings and thus the result holds for Jacobson semisimplicity, nil semisimplicity and nilpotent semisimplicity (semiprime). The results are then applied to semigroup rings. We prove that if $R$ is a commutative ring with identity and $D$ is a commutative semigroup such that a power of each element lies in a subgroup then the semigroup ring $R D$ is semiprime if and only if $D$ is a semilattice of groups $G_{\alpha}, \alpha \in \Omega$, and $R G_{\alpha}$ is semiprime for every $\alpha \in \Omega$. This generalizes Theorem 5.21 of Clifford and Preston [1]. In the paper subsequent to this one Janeski and the author study regularity of semilattice sum decompositions.

2. Preliminaries. Some concepts from the theory of semigroups may be useful to the reader. A band is a semigroup in which every element is idempotent $\left(x^{2}=x\right)$. A semilattice is a commutative band. If $\Omega$ is a semilattice, the natural partial order is defined on $\Omega$ by $e \leqq f$ if and only if $e f=f e=e$.

Received by the editors September 6, 1972.

AMS (MOS) subject classifications (1970). Primary 16A21, 16A48, 16A12, $20 \mathrm{M} 25$.

${ }^{1}$ This research was supported in part by U.S. Air Force Office of Scientific Research Grant AFOSR-72-2164.

(C) American Mathematical Society 1973 
If $R$ is a ring and $R_{\alpha}, \alpha \in \Omega$, a collection of subrings then $\sum_{\alpha \in \Omega} R_{\alpha}=$ $\left\{r_{\alpha_{1}}+\cdots+r_{\alpha_{n}} \mid r_{\alpha_{i}} \in R_{\alpha_{i}}\right\}$.

Definitions. A ring $R$ is a semilattice sum of subrings $R_{\alpha}, \alpha \in \Omega$, if $\Omega$ is a semilattice, $R=\sum_{\alpha \in \Omega} R_{\alpha}$ and $R_{\alpha} R_{\beta} \subseteq R_{\alpha \beta}$.

A ring $R$ is a supplementary semilattice sum of subrings $R_{\alpha}, \alpha \in \Omega$, if $R$ is a semilattice sum of subrings $R_{\alpha}, \alpha \in \Omega$, and if for every $\alpha \in \Omega$, $R_{\alpha} \cap \sum_{\beta \neq \alpha} R_{\beta}=\{0\}$; i.e., if the sum is direct considered as abelian groups under addition.

We shall often use the abbreviated expression, $R=\sum_{\alpha \in \Omega} R_{\alpha}$ is a (supplementary) semilattice sum.

REMARK. The concept of supplementary sum is mentioned in Divinsky [2]. Obviously the concepts of semilattice sum and supplementary semilattice sum can be generalized to band sum and supplementary band sum by removing the condition that $\Omega$ be commutative. If $R$ is a direct sum of ideals $I_{\alpha}, \alpha \in \Omega$, then it is a supplementary semilattice sum of the subrings $I_{\alpha}, \alpha \in \Omega$, for if $\alpha, \beta \in \Omega$ then $I_{\alpha} I_{\beta}=\{0\}$ and therefore multiplication on $\Omega$ can be defined by $\alpha \beta=\alpha_{0}$ where $\alpha_{0}$ is a fixed element of $\Omega$.

If $R=\sum_{\alpha \in \Omega} R_{\alpha}$ is a supplementary semilattice sum and $x=x_{\alpha_{1}}+\cdots+$ $x_{\alpha_{n}} \in R$ with $x_{\alpha_{i}} \in R_{\alpha_{i}}$, then we write $x\left(\alpha_{i}\right)=x_{\alpha_{i}}$. This defines a function $x: \Omega \rightarrow R$. It is well defined since in a supplementary sum the representation of each element is unique. Using this notation we have that if $x, y \in R$ and $\gamma \in \Omega$ then $x y(\gamma)=\sum_{\alpha \beta=\gamma} x(\alpha) y(\beta)$.

Continuing with the assumption that $R=\sum R_{\alpha}$ is a supplementary semilattice sum, we define, for $x \in R, \operatorname{Supp}(x)=\{\alpha \in \Omega \mid x(\alpha) \neq 0\}$ and $\Gamma(x)=\langle\operatorname{Supp}(x)\rangle$ the semigroup generated by $\operatorname{Supp}(x) . \Gamma(x)$ is called the support semigroup of $x$ and is a finite subsemigroup of $\Omega$. Also for $\gamma \in \Omega$, define

$$
R^{\gamma}=\sum_{\alpha \in \Omega ; \alpha \leqq \gamma} R_{\alpha} .
$$

LEMMA 1. If $R=\sum_{\alpha \in \Omega \Omega} R_{\alpha}$ is a supplementary semilattice sum then for every $\gamma \in \Omega, R^{\gamma}$ is an ideal of $R$ and $R_{\gamma}$ is a homomorphic image of $R^{\gamma}$.

Proof. If $\alpha \leqq \gamma$ then $\alpha \beta \leqq \gamma$ for every $\beta \in \Omega$. Hence $R_{\alpha} R_{\beta} \subseteq R_{\alpha \beta} \subseteq R^{\gamma}$. So $R^{\gamma}$ is an ideal.

For $x \in R^{\gamma}$ define $\varphi(x)=x(\gamma)$. Then $\varphi: R^{\gamma} \rightarrow R_{\gamma}$. Clearly $\varphi$ is onto and $\varphi(x+y)=\varphi(x)+\varphi(y)$ for every $x, y \in R^{\gamma}$. If $x, y \in R^{\gamma}$ then $\varphi(x y)=x y(\gamma)=$ $\sum_{\alpha \beta=\gamma} x(\alpha) y(\beta)$. Now $x(\alpha) y(\beta)=0$ if either $\alpha \notin \operatorname{Supp}(x)$ or $\beta \notin \operatorname{Supp}(y)$. But $\alpha \in \operatorname{Supp}(x)$ implies that $\alpha \leqq \gamma$ and $\beta \in \operatorname{Supp}(y)$ implies that $\beta \leqq \gamma$. Hence $x(\alpha) y(\beta) \neq 0$ implies that $\gamma=\alpha \beta \leqq \alpha \leqq \gamma$. We conclude that $\alpha=\gamma$ and similarly $\beta=\gamma$. Therefore $\varphi(x y)=x y(\gamma)=x(\gamma) y(\gamma)=\varphi(x) \varphi(y)$ and hence $\varphi$ is a homomorphism. 
3. $\pi$-semisimplicity. Let $\pi$ be a property of rings. We say $R$ is a $\pi$-ring if it has property $\pi$. An ideal of a ring is said to be a $\pi$-ideal if as a ring it has property $\pi$.

We assume henceforth that the property $\pi$ satisfies: (i) homomorphic images of $\pi$-rings are $\pi$-rings, and (ii) ideals of $\pi$-rings are $\pi$-rings. For example, the properties of being nil, left quasi-regular, locally nilpotent, nilpotent are such properties. A ring is said to be $\pi$-semisimple if it has no $\pi$-ideals.

Leмma 2. Let $R=\sum R_{\alpha}$ be a supplementary semilattice sum where $R_{\alpha}$ is $\pi$-semisimple for every $\alpha \in \Omega$. Suppose $I$ is $a \pi$-ideal of $R$ and let $x \in I$. Then $\operatorname{Supp}(x)$ does not contain a maximum element with respect to the natural partial order on $\Omega$.

Proof. Let $x=x\left(\alpha_{1}\right)+\cdots+x\left(\alpha_{n}\right) \in I$ with $\alpha_{n} \geqq \alpha_{i}, i=1, \cdots, n$. We will derive a contradiction. Since $\alpha_{n} \geqq \alpha_{i}$ for $i=1, \cdots, n$, we have that $x \in R^{\alpha}{ }_{n}$. Let $J=R^{\alpha}{ }_{n} \cap I$. Then $x \in J$ and $J$ is an ideal of $I$ since $R^{\alpha_{n}}$ is an ideal of $R$. Hence $J$ is a $\pi$-ring. Let $\varphi: R^{\alpha_{n}} \cap R_{\alpha_{n}}$ be the epimorphism defined in Lemma 1. Now $J$ is an ideal of $R^{\alpha_{n}}$, so $\varphi(J)$ is a $\pi$-ideal of $R_{\alpha_{n}}$. Since $0 \neq x\left(\alpha_{n}\right) \in \varphi(J), \varphi(J)$ is nonzero. This is the desired contradiction.

The main theorem on semisimplicity is proven for rings which are supplementary semilattice sums of subrings having the following property:

(A) For every $x \neq 0 \in R$, there exist $x^{\prime}, \dot{x}^{\prime \prime} \in R$ such that $0 \neq x x^{\prime}=x x^{\prime} x^{\prime \prime}$.

Any ring with an idempotent which is not a zero divisor satisfies (A).

THEOREM 1. Suppose $R=\sum_{\alpha \in \Omega} R_{\alpha}$ is a supplementary semilattice sum where each $R_{\alpha}$ satisfies condition (A). If $R_{\alpha}$ is $\pi$-semisimple for every $\alpha \in \Omega$ then $R$ is $\pi$-semisimple.

Proof. Suppose $I$ is a $\pi$-ideal of $R$. Choose $x \in I, x \neq 0$, such that $\Gamma(x)$ is minimal. Say $x=x\left(\alpha_{1}\right)+\cdots+x\left(\alpha_{n}\right)$ with $x\left(\alpha_{i}\right) \neq 0, i=1, \cdots, n$. Fix $j$ such that $1 \leqq j \leqq n$ and let

$$
\begin{aligned}
y= & x\left(\alpha_{1}\right) x\left(\alpha_{1}\right)^{\prime}+\cdots+x\left(\alpha_{n}\right) x\left(\alpha_{n}\right)^{\prime} \\
& -x\left(\alpha_{1}\right) x\left(\alpha_{1}\right)^{\prime} x\left(\alpha_{j}\right)^{\prime \prime}-\cdots-x\left(\alpha_{n}\right) x\left(\alpha_{n}\right)^{\prime}\left(\alpha_{j}\right)^{\prime \prime}
\end{aligned}
$$

where $x\left(\alpha_{i}\right)^{\prime}, x\left(\alpha_{1}\right)^{\prime \prime}$ are as hypothesized by condition (A). Now Supp $(y) \subseteq$ $\left\langle\alpha_{1}, \cdots, \alpha_{n}\right\rangle=\Gamma(x)$ so $\Gamma(y) \subseteq \Gamma(x)$. Also $x\left(\alpha_{i}\right) x\left(\alpha_{i}\right)^{\prime} \neq 0$ for $i=1, \cdots, n$ and $x\left(\alpha_{i}\right) x\left(\alpha_{i}\right)^{\prime} x\left(\alpha_{j}\right)^{\prime \prime} \in R_{\alpha_{i} \alpha_{j}}$ so if $y=0$ then for every $i=1, \cdots, n$ there exists $i^{\prime}$ such that $\alpha_{i}^{\prime} \alpha_{j}=\alpha_{i}$. This implies $\alpha_{i} \alpha_{j}=\alpha_{i}$ which says that $\alpha_{j}$ is a maximum element in $\operatorname{Supp}(x)$ contrary to Lemma 2. Therefore $y \neq 0$ and 
by minimality of $\Gamma(x)$ we have $\Gamma(y)=\Gamma(x)$. Hence

$$
\begin{aligned}
& \alpha_{j} \in \Gamma(x)=\Gamma(y) \\
& \quad=\left\langle\alpha_{1}, \cdots, \alpha_{j-1}, \alpha_{j+1}, \cdots, \alpha_{n}, \alpha_{1} \alpha_{j}, \cdots, \alpha_{j-1} \alpha_{j}, \alpha_{j+1} \alpha_{j}, \cdots, \alpha_{n} \alpha_{j}\right\rangle^{\prime \prime}
\end{aligned}
$$

Therefore there exists $k_{j} \neq j$ such that $\alpha_{j}=\alpha_{k_{j}} \alpha_{j}$ or in other words $\alpha_{j}<\alpha_{k_{j}}$. Since the above argument works for every $j=1, \cdots, n$, we can apply it to $k_{j}$ and successively to each greater element that is obtained. Since $\operatorname{Supp}(x)$ is finite we would eventually obtain elements $\alpha_{k}, \alpha_{l}$ such that $\alpha_{k}<\alpha_{l}<\alpha_{k}$. This contradiction establishes the theorem.

In general the converse is not true. Let $\pi$ be the property right-quasi regular. Then $R$ is $\pi$-semisimple if and only if $R$ is Jacobson semisimple. Let $T$ be any integral domain which is not Jacobson semisimple (for example let $T$ be the power series ring in one indeterminate over a field) and let $Q$ be the quotient field of $T$. Let $R$ be the subring of the ring of countably infinite row finite matrices over $Q$ consisting of all matrices $A$ for which there exists $n$ such that, for $i, j>n$,

$$
\begin{array}{ll}
A_{i j}=0, & i \neq j, \\
A_{i j}=a \in T, & \text { if } i=j .
\end{array}
$$

Let $P$ be the ideal of $R$ consisting of all matrices $A$ for which there exists $n$ such that if $i, j>n, A_{i j}=0 . P$ is a prime ideal. Let $S$ be the subring of $R$ consisting of all matrices $A$ for which $A_{i j}=0, i \neq j$ and $A_{i i}=a \in T$ for all positive integers $i, j$. Then $R$ is a supplementary semilattice sum of the subrings $S$ and $P$. But $S \cong T$ so $S$ is not Jacobson semisimple. However $R$ is a primitive ring (see $[4$, p. 36]) so certainly semisimple.

We observe without proof the following: Suppose that $R=\sum_{\alpha \in \Omega} R_{\alpha}$ is a supplementary semilattice sum of subrings $R_{\alpha}$ where each $R_{\alpha}$ has an identity element which lies in the center of $R$. If for every $\alpha \in \Omega$ there are no nonzero nilpotent elements in $R_{\alpha}$, then there are no nonzero nilpotent elements in $R$. The proof is similar to that of Theorem 1 .

4. Semigroup rings. If $D$ is a semigroup and $R$ is a ring, the semigroup ring of $D$ over $R$, written $R D$, is defined to be the set of all functions from $D$ into $R$ which are zero off a finite set. We write elements of $R D$ as finite formal sums $x=r_{1} d_{1}+\cdots+r_{n} d_{n}$ where $x\left(d_{i}\right)=r_{i} \in R$. Addition is pointwise and multiplication is convolution. Thus if $x, y \in R D$ then $(x+y)(d)=$ $x(d)+y(d)$ and $x y(d)=\sum_{a b=d} x(a) y(b)$. Under these operations $R D$ is a ring.

Note that if the semigroup $D$ has a zero $z$ we will not identify $z$ with the zero of the semigroup ring $R D$. Thus $r z=0$ if and only if $r=0$ in $R$. Semigroup rings have been studied in [1], [5], [8] and [10]. 
A semigroup $D$ is a semilattice (band) of semigroups $D_{\alpha}, \alpha \in \Omega$, if $\Omega$ is a semilattice (band), $D$ is a disjoint union of the subsemigroups $D_{\alpha}, \alpha \in \Omega$, and $D_{\alpha} D_{\beta} \subseteq D_{\alpha \beta}$, for all $\alpha, \beta \in \Omega$. Semilattice decompositions of semigroups have been extensively studied (see [1], [6], [7], [9]). Observe that if $D$ is a semilattice of semigroups $D_{\alpha}, \alpha \in \Omega$, then the semigroup ring $R D$ is a supplementary semilattice sum of the semigroup ring $R D_{\alpha}, \alpha \in \Omega$. Thus we have the following corollary to Theorem 1.

Corollary 1. Let $R$ be a ring satisfying condition (A). Suppose D is a semilattice of semigroups $D_{\alpha}, \alpha \in \Omega$, where each $D_{\alpha}$ has an identity. If $R D_{\alpha}$ is $\pi$-semisimple, for every $\alpha \in \Omega$ then $R D$ is $\pi$-semisimple.

Proof. If $R$ satisfies (A) and $D_{\alpha}$ has an identity then $R D_{\alpha}$ satisfies (A).

Corollary 1 applies in particular to the case when $R$ has an identity and $D$ is a semilattice of groups. It would be interesting to know if the converse holds when $D$ is a semilattice of groups. We can answer the question for a wide class of properties, but only in the very special case when the groups have one element; i.e., when $D$ is a semilattice.

LEMMA 3. Suppose $\pi$ is a property such that $R / I$ and I being $\pi$-rings imply that $R$ is a $\pi$-ring. Let $R=\sum_{\alpha \in \Omega} R_{\alpha}$ be a supplementary semilattice sum, where $\Omega$ is a finite semilattice. If each $R_{\alpha}$ is $a \pi$-ring then $R$ is $a \pi$-ring.

Proof. In the following paper [3, Lemma 3] we show that there exist disjoint nonempty subsemilattices $A, B$ of $\Omega$ with $\Omega=A \cup B$ such that $R_{A}=\sum_{\alpha \in A} R_{\alpha}, R_{B}=\sum_{\beta \in B} R_{\beta}$ and $R=R_{A}+R_{B}$ are all supplementary semilattice sums where $A^{2}=A, B^{2}=B=A B=B A$. So an induction argument will complete the proof once we prove the theorem when $|\Omega|=2$. So let $T=T_{\alpha}+T_{\beta}$ be a supplementary semilattice sum. Then since $\{\alpha, \beta\}$ is a semilattice either $\alpha \beta=\beta \alpha=\alpha$ or $\alpha \beta=\beta \alpha=\beta$. Suppose $\alpha \beta=\beta \alpha=\beta$. Then $T_{\beta}$ is an ideal of $T$. Hence $T / T_{\beta} \cong T_{\alpha} / T_{\alpha} \cap T_{\beta}$. Therefore $T / T_{\beta}$ being a homomorphic image of a $\pi$-ring is a $\pi$-ring. Since $T_{\beta}$ is a $\pi$-ring, we conclude that $T$ is a $\pi$-ring. Consider the following condition on properties of rings:

A supplementary semilattice sum of $\pi$-rings $\sum_{\alpha \in \Omega} R_{\alpha}$ over $\Omega$ is a $\pi$-ring if, for every finite subsemilattice $\Omega^{\prime} \subseteq \Omega$, the supplementary semilattice sum $\sum_{\alpha \in \Omega^{\prime}} R_{\alpha}$ is a $\pi$-ring.

The properties of right quasi-regular, nil and regular satisfy (F).

THEOREM 2. Assume $\pi$ satisfies (F) and the hypothesis of Lemma 3. Let $\Omega$ be a semilattice. If the semigroup ring $R \Omega$ is $\pi$-semisimple then $R$ is $\pi$-semisimple. 
Proof. $R \Omega$ is a supplementary semilattice sum of the subrings $R \alpha=$ $\{R \alpha \mid \Omega \in R\}, \alpha \in \Omega$. If $R$ has a $\pi$-ideal $P$, then by Lemma 3 and condition (F), $P \Omega=\sum_{\alpha \in \Omega} P \alpha$ is a $\pi$-ideal of $R \Omega$. Hence $P=\{0\}$.

Corollary 2. Assume $\pi$ is as in Theorem 2. Let $\Omega$ be a semilatice and $R$ a ring with identity. Then $R \Omega$ is $\pi$-semisimple if and only if $R$ is $\pi$ semisimple.

We now prove a generalization of Theorem 5.21 in [1]. If $D$ is a semigroup, $D^{1}$ will denote the semigroup obtained from $D$ by adjoining an identity element. Recall that if a semigroup $D$ has a zero, $r z$ considered as an element of the semigroup ring $R D$ is zero if and only if $r=0$ in the ring $R$.

Lemma 4. Suppose $D$ is an ideal extension of a group $G$ by a commutative nil semigroup. If $R D$ has no nilpotent ideals then $D$ is a group.

Proof. We first show that $D$ is separative $\left(a^{2}=a b=b a=b^{2}\right.$ implies $a=b)$. Let $e$ be the identity of $G$. Then $e d=d e$, for every $d \in D$. Assume that $a, b \in D$ with $a^{2}=a b=b a=b^{2}$. Then $(a e)^{2}=(a e)(b e)=(b e)^{2}$ and $a e, b e \in G$. Hence $a e=b e$. Therefore for every $g \in G, a g=b g$ and similarly $g a=g b$. Since $D / G$ is commutative, then $c d \notin G$ implies $c d=d c$. Now consider $a-b \in R D$. We show that the principal ideal $I$ generated by $a-b$ is nilpotent. In fact, $I^{2}=\{0\}$. It suffices to show that $(a-b) d(a-b)=0$ for all $d \in D^{1}$. If $d=1$, it is clear. Let $y=(a-b) d(a-b)=a d a-b d a-$ $a d b+b d b$. If $a d, b d$ are both in $G$, then $(a d) a=(a d) b$ and $(b d) a=(b d) b$. So $y=0$. Suppose one of $a d, b d$ is not in $G$. Without loss of generality assume $a d \in G$ and $b d \notin G$. Then $(a d) a=(a d) b$ and $(b d) a=d b a=d b^{2}=$ $b d b$. So $y=0$.

A ring is semiprime if it has no nonzero nilpotent ideals. A commutative ring is semiprime if and only if it has no nil ideals.

THEOREM 3. Suppose $R$ is a commutative ring with identity and that $D$ is a commutative semigroup such that a power of each element lies in a subgroup. Then $R D$ is semiprime if and only if $D$ is a semilattice of groups $G_{\alpha}, \alpha \in \Omega$, and $R G_{\alpha}$ is semiprime for every $\alpha \in \Omega$.

Proof. Suppose $R D$ is semiprime. If $D$ is as stated then $D$ is a semilattice of semigroups $D_{\alpha}, \alpha \in \Omega$, where each $D_{\alpha}$ contains a group-ideal $G_{\alpha}$ with $D_{\alpha} / G_{\alpha}$ a nil semigroup (see [1, $\S 4.3$, Exercise 5]). Now if $R D_{\alpha}$ had a nilpotent ideal $N_{\alpha}$, then since $R D$ is commutative, $N_{\alpha}$ would generate a nilpotent ideal of $R D$. Hence, for every $\alpha \in \Omega, R D_{\alpha}$ has no nonzero nilpotent ideal and, by Lemma $4, D_{\alpha}$ is a group. The converse follows from Theorem 1.

Remark. Let $D$ be a union of groups and $F$ a field. In [4], Munn proved that if $D$ is finite then $F D$ semisimple implies $D$ is a semilattice of 
groups. The preceding theorem shows that the same conclusion holds if $D$ is commutative and $F D$ is semiprime. Janeski and Weissglass have proven the identical conclusion under the assumption that $F D$ is regular (without assuming either commutativity or finiteness). One might be led to conjecture that if $D$ is a union of groups and $F D$ is Jacobson semisimple then $D$ is a semilattice of groups. This is false. Let $D=\mathscr{M}\left(G^{0}, 2,2, P\right)$ be the Rees $2 \times 2$ matrix semigroup over the group with zero $G^{0}$ with sandwich matrix $P$ (cf. $[1$, p. 88]) where $G$ is the infinite cyclic group with generator $a$ and

$$
P=\left[\begin{array}{ll}
1 & a \\
1 & 1
\end{array}\right] \text {. }
$$

By applying Theorem 3.8 of [10], $Q D$ is Jacobson semisimple. But $D$ is a union of groups (in fact $D$ is a completely simple semigroup) which is not a semilattice of groups.

Questions. 1. Find a condition on $\Omega$ such that if $R$ is the supplementary semilattice sum of $R_{\alpha}, \alpha \in \Omega$, then $R \pi$-semisimple implies $R_{\alpha}$ is $\pi$-semisimple.

2. If $R=\sum R_{\alpha}, \alpha \in \Omega$, is a supplementary semilattice sum and $R$ is commutative, does $R$ Jacobson semisimple imply that each $R_{\alpha}$ is Jacobson semisimple?

3. Can any of the results in this article be generalized to the case where $\Omega$ is a band?

4. Can condition (A) be removed in Theorem 1 ?

5. Find necessary and sufficient conditions that a ring be a supplementary semilattice sum of division rings.

6. Find conditions to insure that a ring be a supplementary semilattice sum of semilattice sum indecomposable rings. Characterize semilattice sum indecomposable rings.

7. Let $D=\bigcup_{\alpha \in \Omega} G_{\alpha}$ be a semilattice of groups. Does the Jacobson semisimplicity of $R D$ imply the Jacobson semisimplicity of $R G_{\alpha}$ for every $\alpha \in \Omega$ ?

8. Find a property $\pi$ of rings such that if $D$ is any semigroup then $R D$ is a $\pi$-ring if and only if $D$ is a semilattice of groups $G_{\alpha}$ and $R G_{\alpha}$ is a $\pi$-ring, for every $\alpha \in \Omega$.

9. Can the commutativity of $R$ be removed in Theorem 3 ?

The author would like to thank Mohan Putcha for many helpful discussions.

\section{BIBLIOGRAPHY}

1. A. H. Clifford and G. B. Preston, The algebraic theory of semigroups. Vol. I, Math. Surveys, No. 7, Amer. Math. Soc. Providence, R.I., 1961. MR 24 \#A2627.

2. N. J. Divinsky, Rings and radicals, Mathematical Expositions, no. 14, Univ. of Toronto Press, Toronto, 1965. MR 33 \#5654. 
3. J. Janeski and J. Weissglass, Regularity of semilattice sums of rings, Proc. Amer. Math. Soc. 39 (1973), 479-482.

4. N. Jacobson, Structure of rings, rev. ed., Amer. Math. Soc. Colloq. Publ., vol. 37, Amer. Math. Soc., Providence, R.I., 1964. MR 36 \#5158.

5. W. P. Munn, On semigroup algebras, Proc. Cambridge Philos. Soc. 51 (1955), 1-15. MR 16, 561.

6. M. S. Putcha, Semilattice decompositions of semigroups, Semigroup Forum 6 (1973), 12-34.

7. M. S. Putcha and J. Weissglass, A semilattice decomposition into semigroups having at most one idempotent, Pacific J. Math. 38 (1971), 225-228.

8. H. Schneider and J. Weissglass, Group rings, semigroup rings and their radicals, J. Algebra 5 (1967), 1-15. MR 35 \#4317.

9. T. Tamura, Quasi-orders, generalized archimedeanness and semilattice decompositions (submitted).

10. J. Weissglass, Radicals of semigroup rings, Glasgow Math. J. 10 (1969), 85-93. MR 42 \#394.

Department of Mathematics, University of California, Santa Barbara, CALIFornia 93106 